\title{
Proton Beam Writing of Passive Polymer Optical Waveguides
}

\author{
T. C. $\operatorname{Sum}^{a}$, A. A. Bettiol ${ }^{a}$, S. Venugopal Rao ${ }^{a}$, J. A. van $\operatorname{Kan}^{a}$, A. Ramam ${ }^{b}$ and F. $\operatorname{Watt}^{a}$ \\ ${ }^{a}$ Centre for Ion Beam Applications (CIBA), 2 Science Drive 3, Singapore 117542 \\ ${ }^{b}$ Institute of Materials Research and Engineering (IMRE), 3 Research Link, Singapore 117602
}

\begin{abstract}
Proton beam writing is a new direct-write micromachining technique capable of producing 3-dimensional (3D), high aspect ratio micro-structures with straight and smooth sidewalls. It uses a focused sub-micron beam of $2.0 \mathrm{MeV}$ protons to direct-write on a suitable polymer, such as the photoresists: poly-methylmethacrylate (PMMA) and $S U-8$, a negative tone photoresist from MicroChem. In this paper, we report on the application of proton beam writing to fabricate low-loss passive polymer waveguide structures such as symmetric y-branching waveguides in SU-8. SU-8 channel waveguides are fabricated by first direct-writing the pattern using a proton beam and subsequently chemically developing the latent image formed. A UV-cured resin, Norland Optical Adhesive $88(N O A-88)$ is used as the cladding layer. Being a direct-write technique, proton beam writing offers us great flexibility to fabricate waveguides of arbitrary patterns and this is an asset that can be applied to the rapid prototyping of optical circuits. With all its unique characteristics, proton beam writing is an excellent technique for waveguide fabrication.
\end{abstract}

Keywords: proton beam writing, polymer, waveguide, SU-8, direct-write, refractive index, prism coupler

\section{INTRODUCTION}

Polymers possesses many unique characteristics such as their high optical clarity, low attenuation, and their excellent weathering ability present us with an interesting class of materials to work with. Its widespread use ${ }^{1}$ has been attributed to the ease of processing and fabrication of polymers. In contrast to inorganic materials such as $\mathrm{LiNbO}_{5}$ and $\mathrm{InP}$, polymeric optical waveguides offer an inexpensive solution for many telecommunication applications. Some common techniques used for polymeric waveguide fabrication include ion implantation with a mask, ${ }^{2,3}$ photolithography and reactive ion etching (RIE) ${ }^{4,5}$ and photo-bleaching. ${ }^{6,7}$ These methods either involve numerous processing steps or require long fabrication times. Direct-write techniques such as electron beam lithography ${ }^{8,9}$ and proton beam writing, ${ }^{10,11}$ on the other hand, have the advantage of being maskless, allowing rapid and inexpensive prototyping. Unlike electron beam exposures, where the electron trajectories are erratic due to multiple large angle collisions, $2.0 \mathrm{MeV}$ protons have a straight trajectory and a very well-defined range in a photoresist (approximately $62 \mu \mathrm{m}$ in SU-8).

In this paper, we report on the application of proton beam writing, a new lithographic tool, to fabricate low-loss passive polymer waveguide structures such as symmetric y-branching waveguides in SU-8. Proton beam writing is a direct-write micromachining technique capable of producing 3-dimensional (3-D), high aspect ratio micro-structures with straight and smooth sidewalls. ${ }^{12}$ It uses a focused sub-micron beam of $2.0 \mathrm{MeV}$ protons to direct-write on a suitable polymer, such as photoresists: poly-methylmethacrylate (PMMA), GS233, a positive tone photoresist from Röhm; and SU-8, a negative tone photoresist from MicroChem Corporation (MCC). The protons were focused using magnetic quadrupole lenses. ${ }^{13}$ Sub-micron beam resolution is extremely critical for proton beam writing as it is important to direct-write the optical pathways accurately in order to realize the optimal design and to minimize optical losses. In the case of a symmetric y-branch, this is crucial for the S-bend segment near the intersection with the linear taper segment. With its own scanning software where a variety of design file formats are supported (such as Autocad ${ }^{T M} *$.dxf), proton beam writing can be used to fabricate

Further author information: (Send correspondence to T. C. Sum)

T. C. Sum: E-mail: scip1089@nus.edu.sg, Telephone: +65 6874 4953, Address: Centre for Ion Beam Applications (CIBA), Department of Physics, National University of Singapore, 2 Science Drive 3, Singapore 117542.

A. A. Bettiol: E-mail: phybaa@nus.edu.sg, Telephone: +65 68744138

CIBA Website: http://www.ciba.nus.edu.sg/ 
any arbitrary waveguide pattern (e.g. symmetric y-branching waveguides or mach-zehnder interferometers) that is exported from commercially available optoelectronic computer aided design (CAD) software such as BeamProp ${ }^{T M}$ from Rsoft Incorporated.

\section{EXPERIMENTAL}

SU-8, is a negative, epoxy-type, chemically-amplified, near-UV photoresist based on EPON SU-8 epoxy resin which is a multifunctional glycidyl ether derivative of bisphenol-A novolac available from Shell Chemical. It has been originally developed and patented by IBM. ${ }^{14}$ Upon exposure to actinic radiation (e.g. ultraviolet, electron beam, X-rays or protons), it forms a ladder-like structure with a high cross-linking density and exhibits good chemical and thermal stability $\left(T_{g}>200^{\circ} C\right)$. SU-8, $\sim 5 \mu m$ thick, was first spin-coated on a suitable glass substrate. The transmission spectra of the cross-linked SU-8 resist was measured using a Shimadzu UV-1601 $U V$-Visible spectrophotometer for the wavelength range of $300 \mathrm{~nm}-1100 \mathrm{~nm}$ and a Shimadzu UV-3101PC $U V$-VIS-NIR spectrophotometer for the wavelength range of $1100 \mathrm{~nm}-1700 \mathrm{~nm}$. The refractive index of the proton beam cross-linked SU-8 was measured using a Metricon Corporation Model 2010 prism coupler.

\subsection{Refractive Index Measurements}

Accurate modeling of optical waveguides is essential to the fabrication of low-loss optical waveguide structures such as symmetric y-branch. One of the important input parameters for the beam propagation method (BPM) software, BeamProp ${ }^{T M}$, is the refractive index of the respective components of the waveguide. The bulk refractive indices of the substrates and the refractive indices of SU-8 and NOA- 88 were measured using a prism coupler. The prism coupler comprises of three major component modules - an optics module, an interface module with attached control pod and a personal computer for data acquisition. It is essential to determine the refractive index of proton beam cross-linked SU-8 as proton beam writing is a unique technique that does not involve the post-exposure bake of the photoresist. To determine the refractive indices of the cross-linked SU-8 resist, a small square of area $2 \times 2 \mathrm{~mm}^{2}$ was first irradiated with $2.0 \mathrm{MeV}$ protons and a dose of $30 \mathrm{nC} / \mathrm{mm}^{2}$. Chemical development of this sample was subsequently carried out to remove the areas not cross-linked by the proton beam. A detailed description of the development procedure will be described in a later section.

The polymer film to be measured is brought into contact with the base of a rutile prism, leaving an air gap of the order of $0.1 \mu \mathrm{m}$. A light beam from a laser enters the prism and strikes the base of the prism. The light is thus totally reflected at the prism base onto the photodetector. The total reflection leaves only evanescent fields in the air gap. Through these evanescent fields, the light wave from the prism is coupled into the waveguide. The prism, the sample and the photodetector are mounted on a rotatory table so that the incident angle of the laser beam can be changed correspondingly. Coupling is strongest when the following phase matching condition is satisfied ${ }^{15}$ :

$$
\beta_{m}=k_{o} n_{p} \sin \left(\theta_{m}\right)
$$

where $\beta_{m}$ is the propagation constant, $k_{o}=w / c, n_{p}$ is the prism refractive index and $m$ is the coupling angle.

At certain angles of incidence, sharp reflectivity dips (as shown in Figure 1), occur in the spectrum corresponding to the excitation of guided modes. This is known as the dark mode line spectrum and the dips are known as the 'dark' m-lines. At $\beta_{m}$, the light is coupled into the waveguide, thus resulting in a lack of reflected light at the base of the prism, consequently forming the dark mode line spectrum as shown. From the positions of $\beta_{m}$, it is possible to determine the mode effective indices, the waveguide thickness, and the refractive index, $n$, of the waveguide. As the minimum beam spot size of the laser is approximately $1 \mathrm{~mm}^{2}$, the $4 \mathrm{~mm}^{2} \mathrm{SU}-8$ square is sufficiently big to be measured. Care must be taken to align the resist to the 'wet-spot' of the prism before clamping it to the prism. Prior to this refractive index measurement of the thin film, the bulk refractive index of the substrate was first determined in a separate measurement. The measured refractive indices of the respective components of the waveguide are given in Table 1. 


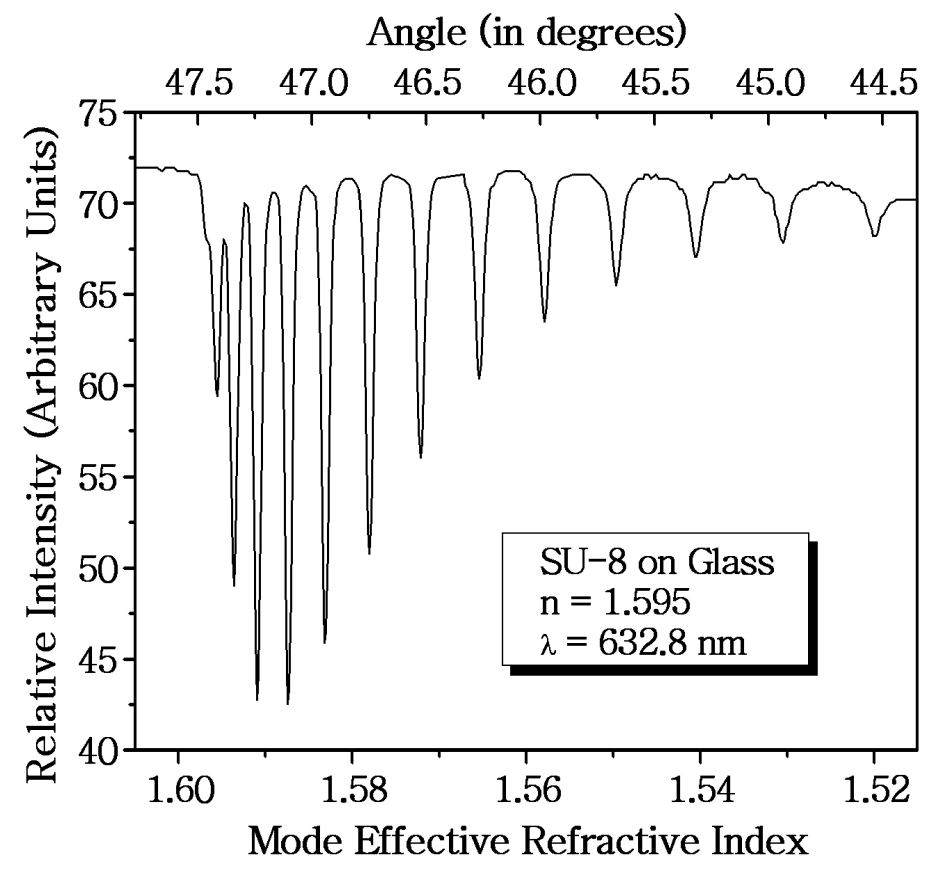

Figure 1. The measured relative intensity of the reflected light from the prism against the effective refractive index of the observed dark modes for the proton beam cross-linked SU-8 at a dose of $30 \mathrm{nC} / \mathrm{mm}^{2}$.

Table 1. Table showing the refractive indices of the glass substrate, the core (SU-8) and the cladding (NOA- 88 UV adhesive).

\begin{tabular}{|c|c|}
\hline Material & Refractive Index $(\mathbf{6 3 2 . 8} \mathbf{~} \mathrm{m})$ \\
\hline Glass Substrate & 1.514 \\
\hline SU-8 (Core) & 1.595 \\
\hline NOA-88 (Cladding) & 1.555 \\
\hline
\end{tabular}

\subsection{Y-Branching Waveguide Design and Fabrication}

The optical y-branching waveguide is one of the elementary structures for integrated optics. It has applications as either an optical power splitter or an optical power combiner. In this paper, the aim is to realize a 3-dB type of power splitter based on a symmetric y-branching waveguide. A cross-sectional view of the waveguide design is shown in Figure 2.

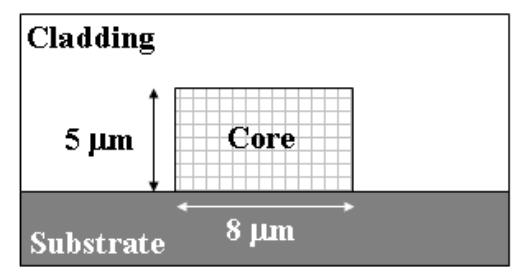

Figure 2. A schematic illustration of the waveguide cross-section. 
As shown in Figure 3, the y-branching waveguide comprises of four segments (from right to left): linear segment, linear taper segment, Cosine S-bend segment and a linear segment.

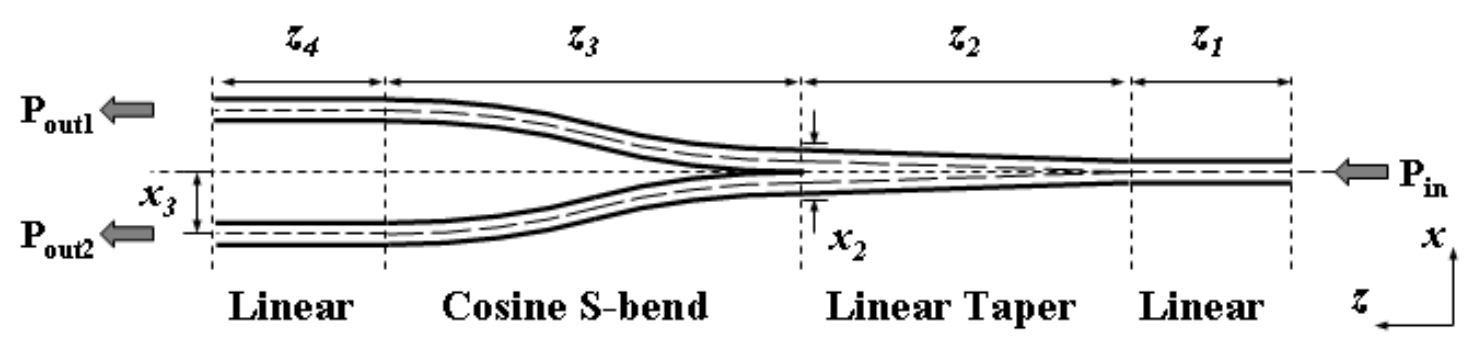

Figure 3. A schematic showing the various segments of the symmetric y-branching waveguide.

The coupled power $P_{i n}$ in the input waveguide is divided in the output arms with the output power $P_{\text {out } 1}$ and $P_{\text {out } 2}$. The functionality of such a y-branching waveguide solely arises from its symmetry. ${ }^{16}$ If the fundamental mode at the input linear segment is excited, it will split evenly between the two arms and the optical power $\left(P_{\text {out } 1}\right.$ and $\left.P_{\text {out } 2}\right)$ exiting the two arms will be equal. Since the splitting action of the y-branching waveguide is dependent only on the symmetry, it is a wavelength-independent $3 d B$ splitter.

The linear tapered segment not only allows for the smooth expansion of the guide width without mode conversion, but also suppresses the occurrence of the first order lateral mode at the branching point. ${ }^{17} \mathrm{~A}$ more compact y-branching waveguide results with the introduction of some curvature in the arms in the form of an S-bend segment as shown in Figure 3. If such S-bend segments were replaced by linear segments, such a waveguide design would be arbitrarily long. ${ }^{16}$ The types of S-bend structures investigated in this paper include the arc type S-bend, Cosine type S-bend and the raised Sine S-bend.

For the design of a low-loss y-branching waveguide, one has to investigate the dependence of the type of S-bend and the branch length, $z_{3}$. The BPM software BeamPROP ${ }^{T M}$ was used for the calculation of the power distribution and the simulation. The refractive indices of the various waveguide components found from prism coupling measurements were input into the simulation program. The cross-section of the channel waveguide is $5 \mu m \times 8 \mu m$ (height $\times$ width). From the parameter scans of the type of S-bend and the branch length, $z_{3}$, optimized designs for y-branching waveguides were obtained. Table 2 gives an overview of the two types of y-branching waveguides that were designed. As shown in Figure 4, for the Type II y-branching waveguide, the Cosine type S-bend with a branch length of $1400 \mu \mathrm{m}$ gives the lowest branching loss and the largest output power in its arms. Figure 5 shows a simulated 3-D plot of the optical power distribution in the y-branch. The optimized designs were subsequently exported to a suitable file format for proton beam writing.

Table 2. Table showing the parameters used in the fabrication of the two different types of y-branching waveguides.

\begin{tabular}{|l|c|c|}
\hline \multicolumn{1}{|c|}{ Segment } & $\begin{array}{c}\text { Type I } \\
\text { Branch Separation }\end{array}$ & $\begin{array}{c}\text { Type II } \\
\mathbf{1 9 0} \mu \mathrm{m} \text { Branch Separation }\end{array}$ \\
\hline Linear & $z_{1}=350 \mu \mathrm{m}$ & $z_{1}=175 \mu \mathrm{m}$ \\
\hline Linear Taper & $x_{2}=16 \mu \mathrm{m} ; z_{2}=350 \mu \mathrm{m}$ & $x_{2}=16 \mu \mathrm{m} ; z_{2}=350 \mu \mathrm{m}$ \\
\hline Cosine S-bend & $x_{3}=22.5 \mu \mathrm{m} ; z_{3}=1000 \mu \mathrm{m}$ & $x_{3}=95 \mu \mathrm{m} ; z_{3}=1400 \mu \mathrm{m}$ \\
\hline Linear & $z_{4}=300 \mu \mathrm{m}$ & $z_{4}=75 \mu \mathrm{m}$ \\
\hline
\end{tabular}




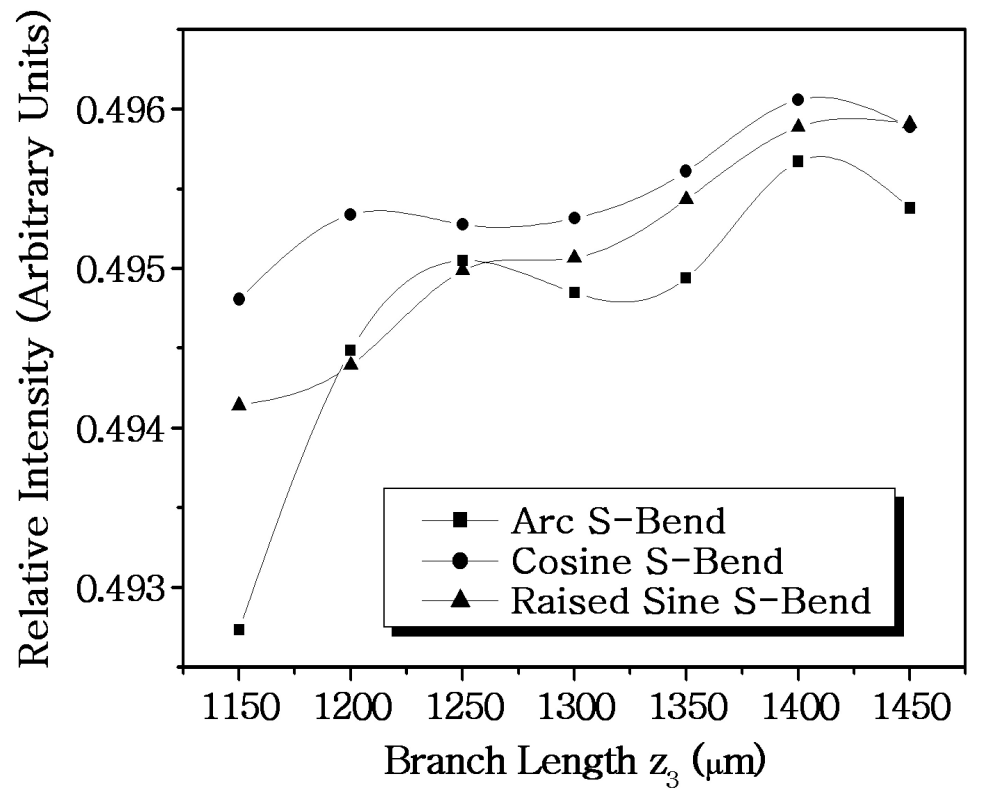

Figure 4. Simulated data on the dependence of the type of S-bend versus the branch length $z_{3}$ for Type II y-branching waveguides.

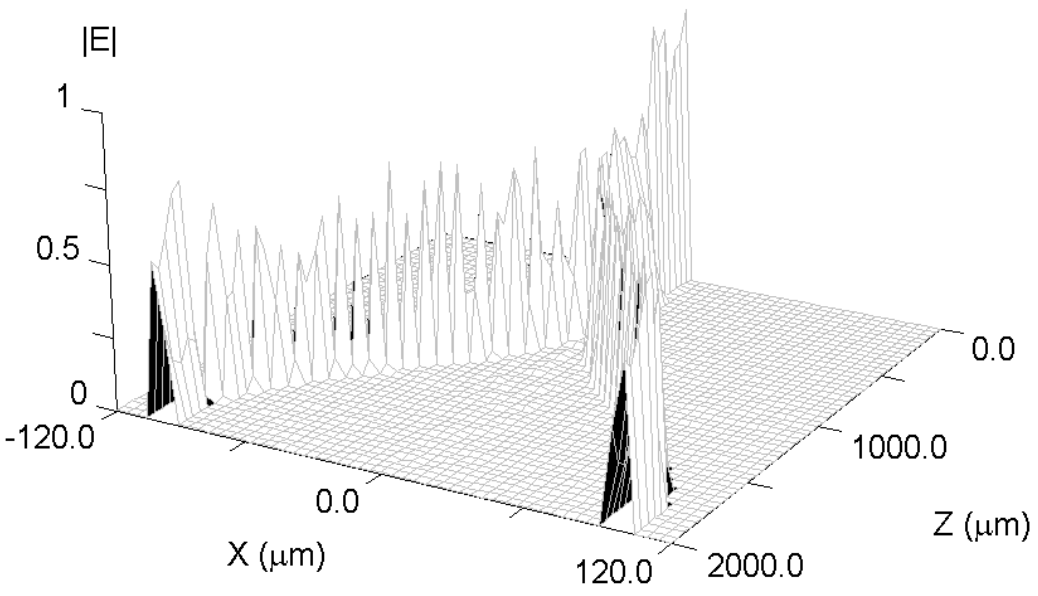

Figure 5. 3-D plot of the power distribution in the y-branching waveguide (Type II) in wire mesh format. 
Proton beam writing was carried out using proton beams from the High Voltage Engineering Europa (HVEE) $3.5 \mathrm{MeV}$ Singletron ${ }^{T M}$ accelerator at the Centre for Ion Beam Applications (CIBA). ${ }^{18}$ The waveguides were direct-written using a $2.0 \mathrm{MeV}$ beam of protons with a beam spot size of $\sim 0.5 \mu \mathrm{m}$ and a beam current of $\sim 2$ $p A$. Symmetric y-branch waveguides were fabricated by magnetically scanning the proton beam over the SU-8 photoresist. A description of the scanning system and the Rutherford backscattering spectrometry (RBS) dose normalization procedure can be found elsewhere. ${ }^{19}$ An optimum dose of $30 \mathrm{nC} / \mathrm{mm}^{2}$ (i.e. $\sim 1.875 \times 10^{13}$ particles $/ \mathrm{cm}^{2}$ ) was used to direct-write the SU-8 waveguides. The $2.0 \mathrm{MeV}$ protons penetrate the $\sim 5 \mu \mathrm{m}$ thick SU-8 resist layer, stopping well into the substrate. A schematic of the SU-8 waveguide fabrication procedure is shown in Figure 6.

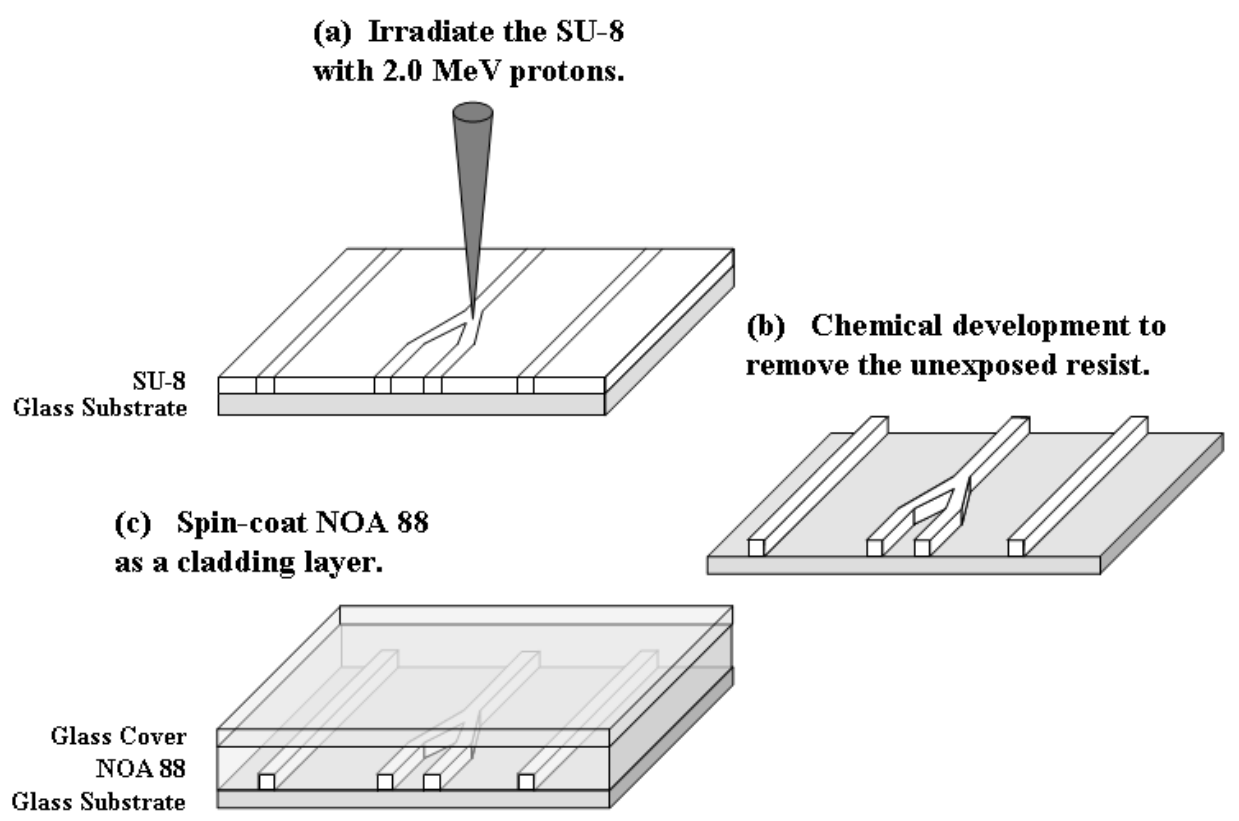

Figure 6. A schematic showing the fabrication procedure of optical waveguides in SU-8 using proton beam writing.

No post-baking of the SU-8 photoresist is needed after proton beam writing. The resist was chemically developed (i.e. the areas not irradiated are chemically removed) in propylenglygol-monoethylether-acetate (PGMEA) for 90 seconds and then rinsed in deionised water. Subsequently, a second rinse of the resist in PGMEA was performed for 30 seconds and followed by a final rinse in fresh deionised water. Channel waveguides with straight and smooth sidewalls, as shown in Figure 9(a), are formed. NOA-88, $30 \mu \mathrm{m}$ thick was then spin-coated over these waveguides to form the cladding layer. In this same step, a piece of glass slide may also be bonded with the NOA- 88 to form a protective cover. The NOA- 88 cladding was subsequently cured for 5 minutes using UV light at $365 \mathrm{~nm}$ wavelength and $200 \mathrm{~W}$ power. The waveguide sample was then baked at $50^{\circ} \mathrm{C}$ for 12 hours in an oven to improve the cladding's adhesion to the glass surfaces.

After baking the sample, the sample was edge-polished until the end faces of the waveguides were exposed with a remaining roughness of less than $60 \mathrm{~nm}$. The waveguides were then end-coupled with monochromatic light $(632.8 \mathrm{~nm})$ using a single mode optical fibre (3M single mode fiber FS-SN-3224, core $\sim 4 \mu \mathrm{m}$ ). Index matching oil from Nye Optical Products $(N C F-446, \mathrm{n}=1.46)$ was applied between the waveguide end-faces and the optical fibre to minimize the light scattering from the waveguide surface. The output light was imaged with a charged coupled device $(\mathrm{CCD})$ camera mounted on a microscope with a $50 \times$ long working distance microscope objective lens $($ N.A. $=0.55)$. The images were obtained in a 16 -bit mode (i.e. a resolution of 65536 gray levels). 


\section{RESULTS AND DISCUSSION}

Figure 7 shows the transmission spectra of cross-linked SU-8. Beyond a wavelength of $400 \mathrm{~nm}$, the transmission is greater than $95 \%$, indicating that SU-8 is optically transparent at $632.8 \mathrm{~nm}$ as well as at the telecommunications wavelengths of $1330 \mathrm{~nm}$ and $1550 \mathrm{~nm}$. Figure 8 shows a top view of one of the $2 \mathrm{~mm}$ long SU- 8 waveguide with a branch separation of $190 \mu \mathrm{m}$ (Type II). Figure 9(a) shows a scanning electron microscope (SEM) micrograph of the SU-8 waveguide prior to the spin-coating of the cladding layer. Note the straight and smooth sidewalls of the proton beam written waveguide. The ability to direct-write waveguides with straight and smooth sidewalls is one of the unique characteristics of proton beam writing. This is crucial to minimizing scattering losses in waveguides. Previously, a propagation loss of approximately $0.19 \pm 0.03 \mathrm{~dB} / \mathrm{cm}$ at $632.8 \mathrm{~nm}$ wavelength for linear SU-8 waveguides has been reported. ${ }^{20}$ A cross-sectional view of the same type of waveguide is shown in Figure 9(b). The waveguide core is approximately $5 \mu m \times 8 \mu m$ (height $\times$ width).

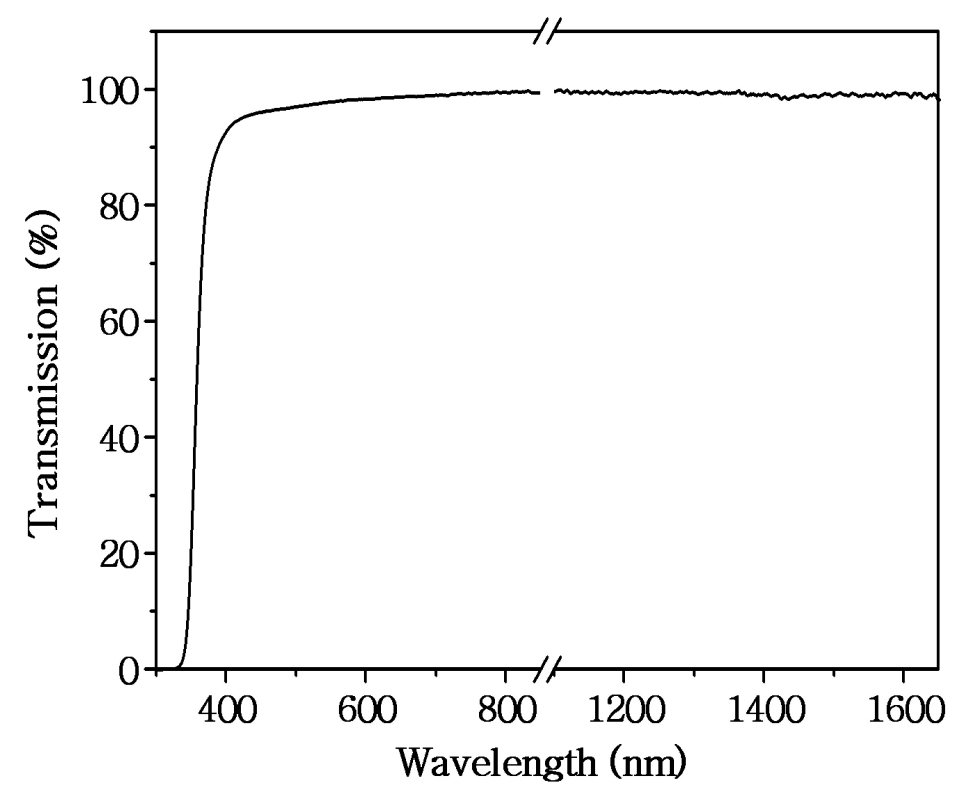

Figure 7. A graph showing the transmission spectra versus wavelength of the cross-linked SU-8 resist. Only the data from the visible wavelengths $(400$ to $800 \mathrm{~nm}$ ) and the communications wavelengths (1330 $\mathrm{nm} \& 1550 \mathrm{~nm})$ are presented.

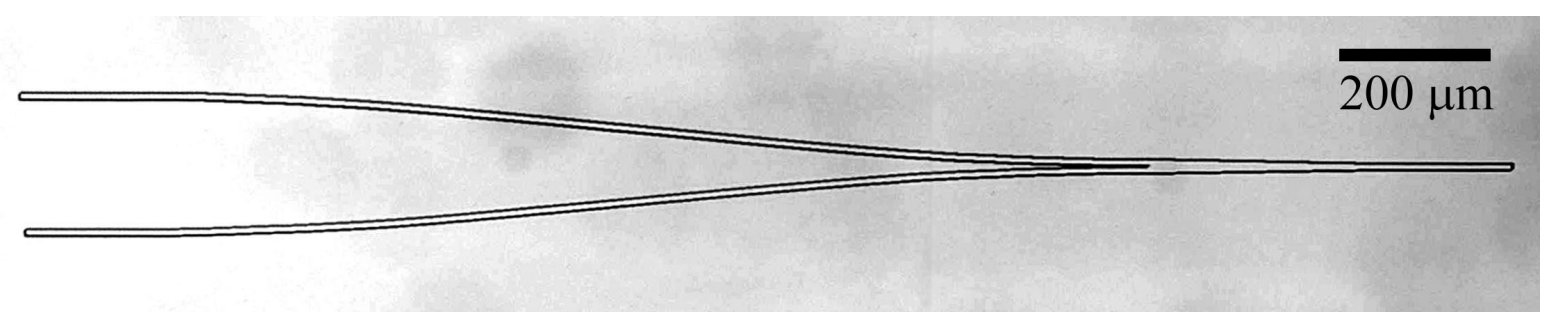

Figure 8. Differential interference contrast (DIC) micrograph of a $2 \mathrm{~mm}$ long symmetric y-branching waveguide (Type II). 


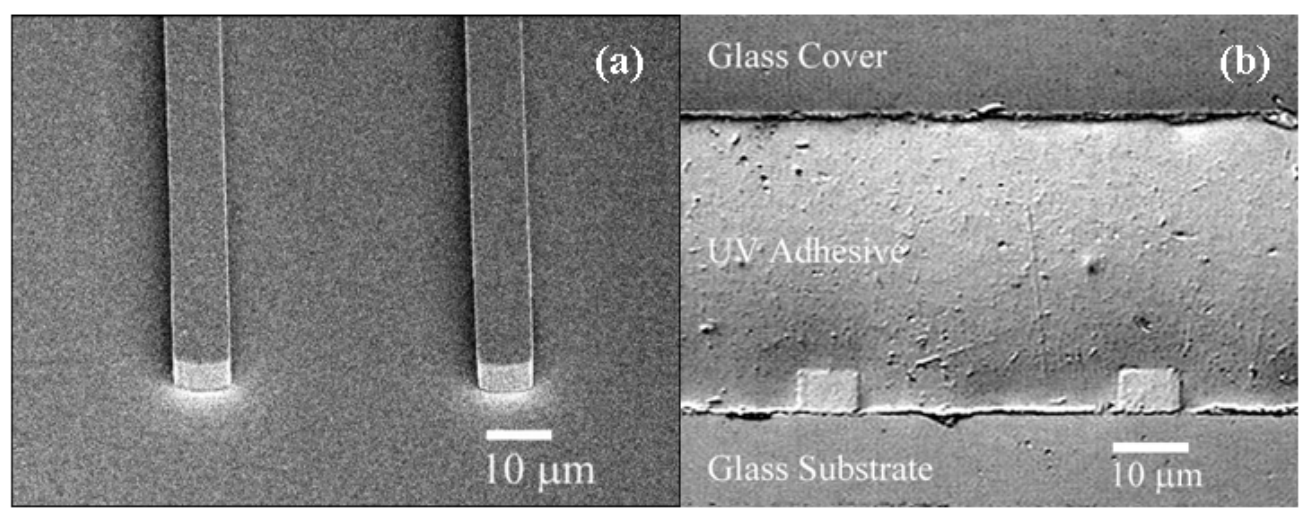

Figure 9. (a) SEM micrograph of the y-branches of a symmetric y-branching waveguide (Type I). (b) DIC micrograph of the cross-sectional view of the same type of SU-8 y-branching waveguide. For added protection, a top glass cover is sometimes bonded with the UV adhesive.

Figure 10(a) shows a near field image of a SU-8 y-branching waveguide (Type I). A 3-D plot of the normalized intensity distributions of the two branches shown in Figure 10(a) is given in Figure 10(b). The ratio of the intensity distributions from each branch was found to be $0.54 / 0.46$.

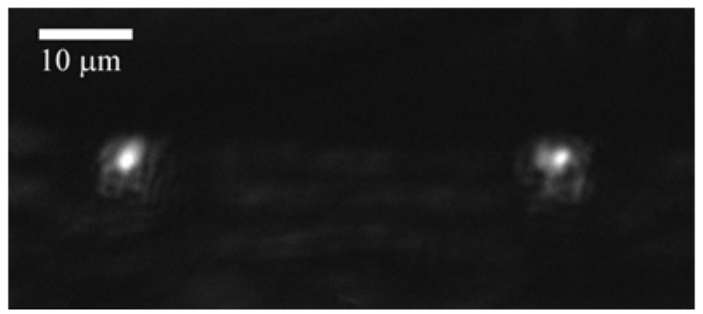

(a)

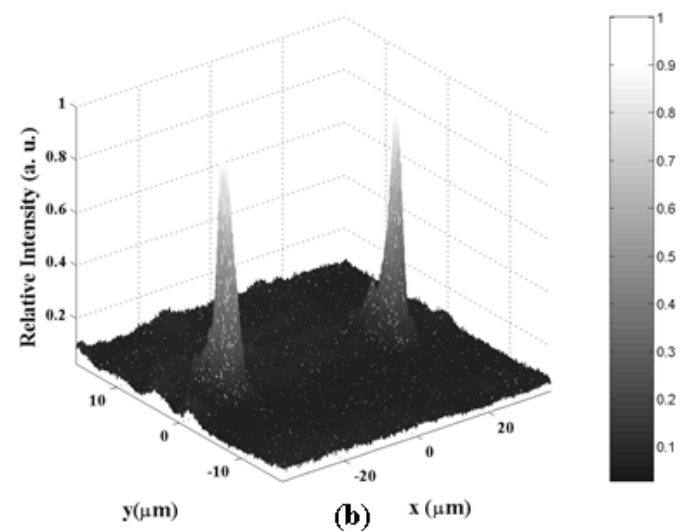

Figure 10. (a) An optical micrograph of the cross-sectional view of a symmetric y-branching SU-8 waveguide (Type I) showing $632.8 \mathrm{~nm}$ light emitting from the branches. (b) A 3-D plot of the normalized intensity emitting from the branches. The ratio of the intensity distribution from the each branch is $0.54 / 0.46$. 


\section{CONCLUSION}

In conclusion, we have applied a new lithographic technique, proton beam writing, to fabricate symmetric ybranching waveguides in SU-8. Waveguides of any arbitrary shape and design can be fabricated using this technique. Earlier results have shown that the linear SU-8 waveguides fabricated using this method exhibit very low propagation loss. ${ }^{20}$ However, as in the case for any direct-write technique compared with masked lithography, the manufacturing throughput from proton beam writing is expected to be low. Nonetheless, proton beam writing offers us great flexibility to fabricate waveguides of arbitrary patterns and this is an asset that can be applied to the rapid prototyping of optical circuits. The shortcoming of this technique could be overcome with the implementation of post-lithographic processes such as electroplating and imprinting, which offers the possibility of fabricating metallic stamps and moulds that can be used for imprinting waveguides. In this manner, the batch production of optical waveguide components and optical circuits would be feasible.

\section{ACKNOWLEDGMENTS}

A Defence Innovative Research Programme (DIRP) grant from the Defence Science and Technology Agency (DSTA), Singapore, supported this work.

\section{REFERENCES}

1. R. Chen, "Integration goes plastic," Oe magazine - The monthly publication of SPIE: The International Society for Optical Engineering 2(11), pp. 24-26, 2002.

2. D. M. Ruck, S. Brunner, K. Tinschert, and W. F. X. Frank, "Production of buried waveguides in pmma by high energy ion implantation," Nucl. Instrum. Methods Phys. Res. B 106, pp. 447-451, 95.

3. W. Hong, H. J. Woo, H. W. Choi, Y. S. Kim, and G. D. Kim, "Optical property modification of pmma by ion-beam implantation," Appl. Surf. Sci. 169-170, pp. 428-432, 2001.

4. M. Hikita, Y. Shuto, M. Amano, R. Yoshimura, S. Tomaru, and H. Kozawaguchi, "Optical intensity modulation in a vertically stacked coupler incorporating electro-optic polymer," Appl. Phys. Lett. 63, pp. 1161-1163, 1993.

5. W. Wang, D. Chen, H. R. Fetterman, Y. Shi, W. H. Steier, and L. R. Dalton, "Travelling wave electro-optic phase modulator using cross-linked nonlinear optical polymer," Appl. Phys. Lett. 65, pp. 929-931, 1994.

6. M. B. J. Diemeer, F. M. M. Suyten, E. S. Trommel, A. McDonach, J. M. Copeland, L. W. Jenneskens, and W. H. G. Horsthuis, "Photoinduced channel waveguide formation in nonlinear optical polymers," Electron. Lett. 26, pp. 379-380, 1990.

7. Y. Shi, W. H. Steier, L. Yu, M. Chen, and L. R. Dalton, "Large stable photoinduced refractive index change in a nonlinear optical polyester polymer with disperse red side groups," Appl. Phys. Lett. 58, pp. 1131-1133, 1991.

8. H. Nakayama, O. Sugihara, and N. Okamoto, "Nonlinear optical waveguide fabrication by direct electronbeam irradiation and thermal development using a high $t_{g}$ polymer," Appl. Phys. Lett. 71, pp. 1924-1926, 1997.

9. W. H. Wong, J. Zhou, and E. Y. B. Pun, "Low-loss polymeric optical waveguides using electron-beam direct writing," Appl. Phys. Lett. 78, pp. 2110-2112, 2001.

10. F. Watt, J. A. van Kan, and T. Osipowicz, "Three-dimesional microfabrication using maskless irradiation with mev ion beams: Proton beam micromachining," MRS Bull. 25(2), pp. 33-38, 2000.

11. T. C. Sum, A. A. Bettiol, H. L. Seng, I. Rajta, J. A. van Kan, and F. Watt, "Proton beam writing of passive waveguides in pmma," Nucl. Instrum. Methods Phys. Res. B 210, pp. 266 - 271, 2003.

12. J. A. van Kan, A. A. Bettiol, B. S. Wee, T. C. Sum, S. M. Tang, and F. Watt, "Proton beam micromachining: a new tool for precision three-dimensional microstructures," Sens. Actuators A 92, pp. 370-374, 2001.

13. G. W. Grime and F. Watt, "Focusing high-energy ion beams," in Principles and Application of High-Energy Ion Microbeams, F. Watt and G. W. Grime, eds., pp. 79-112, IOP Publishing Ltd, England, 1987.

14. J. M. Shaw, J. D. Gelorme, N. C. LaBianca, W. E. Conley, and S. J. Holmes, "Negative photoresists for optical lithography," IBM J. Res. Develop. 41(1/2), pp. 81-94, 1997. 
15. R. Ulrich and R. Torge, "Measurement of thin film parameters with a prism coupler," Appl. Opt. 12(12), pp. 2901-2908, 1973.

16. F. Ladouceur and J. D. Love, Silica-based Buried Channel Waveguides and Devices, Chapman \& Hall, London, 1996.

17. H. Nishihara, M. Haruna, and T. Suhara, Optical Integrated Circuits, McGraw-Hill Book Company, New York, 1989.

18. F. Watt, J. A. van Kan, I. Rajta, A. A. Bettiol, T. F. Choo, M. B. H. Breese, and T. Osipowicz, "The national university of singapore high energy ion nano-probe facility: Performance tests," Nucl. Instrum. Methods Phys. Res. B (210), pp. 14-20, 2003.

19. A. A. Bettiol, J. A. van Kan, T. C. Sum, and F. Watt, "A labview ${ }^{T M}$-based scanning and control system for proton beam micromachining," Nucl. Instrum. Methods Phys. Res. B (181), pp. 49-53, 2001.

20. T. C. Sum, A. A. Bettiol, J. A. van Kan, F. Watt, E. Y. B. Pun, and K. K. Tung, "Proton beam writing of low-loss polymer optical waveguides," Appl. Phys. Lett. 83(9), pp. 1707-1709, 2003. 